\title{
Anesthetic Management of Traumatic Rupture of Aorta and Diaphragm at Shahid Gangalal National Heart Centre
}

\author{
Upreti MR*, Pokhrel JN* \\ *Shahid Gangalal National Heart Centre \\ Corresponding: Dr. Dr.Murari Raj Upreti \\ Shahid Gangalal National Heart Centre, Bansbari, Kathmandu, Nepal Tel: 0097-1-4371322, 00977-1-437137 Fax 00977-1-4371123
}

\begin{abstract}
Traumatic transaction thoracic aorta and rupture diaphragm carries high mortality rate. It needs specialized centre which is able to handle such case and prompt intervention. Even with maximum effort, only 5 to $7 \%$ of the victim will survive and get discharged from the hospital.
\end{abstract}

Keywords : aorta, diaphragm, shock, hypoxia

\section{INTRODUCTION}

Blunmt thoracic aortic injury is the secont most common cause of death after severe head injury in trauma victims. It is always associated with other injuries. $85 \%$ of all the victims die on the scene of accident, and half of those who are taken to the hospital die within 24 hours or during transfer.

The most common site of injury is aortic isthmus, just below the origin of the left subclavien artery, because aortic arch is relatively mobile and descending aorta is fixed.

There are two mechanisms involved in blunt traumatic injury of the thoracic aorta. One is due to deferential decleration of the aorta while fast moving automobile stops suddenly. So there is $t=o r s i o n$ force, differential of the aortic segment and hydrostatic force and various combination of this mechanism will be involved during blunt trauma.

Second is the *osseous pinch "mechanism where there is compression of aorta between manuubrium, clavicle, first rib and spine.

These patients are always associated with other injuries such as treaumatic rupture of aortic valve, rupture of the diaphragm, head injury, facial injury, pelvic fracture etc.

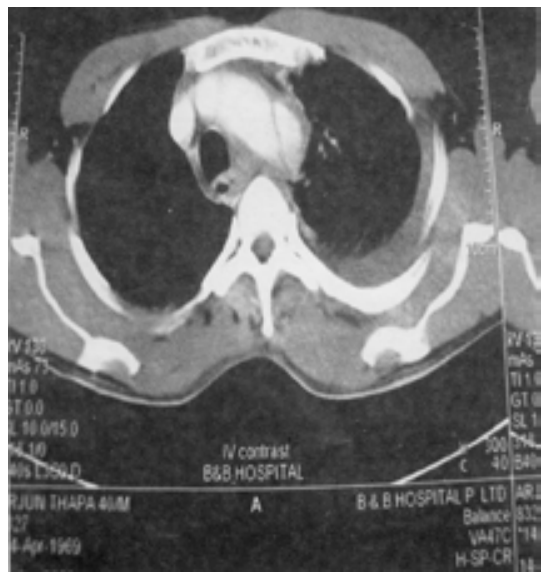

Figure 1: CT scan showing transaction of descending aorta below the original left subclavian artery

\section{CASE REPORT}

40 years man of approximately $70 \mathrm{~kg}$, sustained automobile accident, and presented to out emergency department with complaints of severe chest pain anteriorly and in the back between the scapula, shortness of breath and tachycardia, approximately after 4 hours of accident.

He was dyspnoeic, had absent breath sound in the left chest, tachycardia at 120 to 1130 beats/minute and a normal blood pressure. Chest $\mathrm{x}$-ray, PA view showed widened mediastinal and abdominal content in the left chest. CBC and hemogram was within normal limits but liver enzymes were slightly elevated, coagulation profile was within normal limit and renal function was not impaired.

Contrast CT scan showed rupture of diaphragm on left side and transaction descending aorta below the original left subclavin artery.. then he was referred to SGNHC.

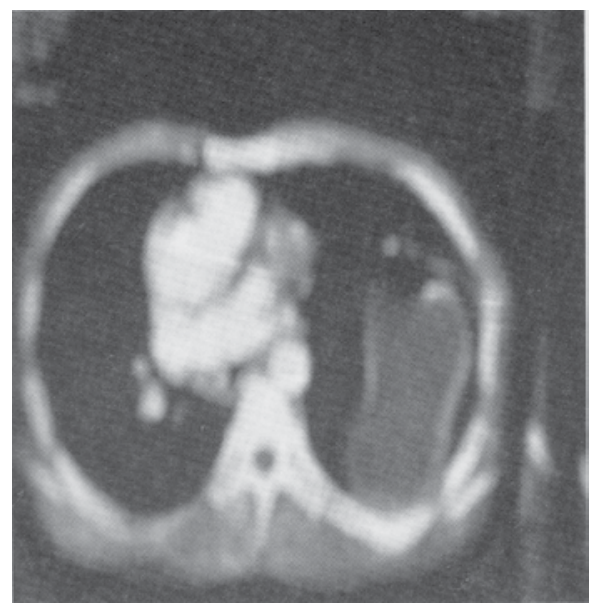

Figure 2: CT scan showing abdominal content in the left chest

He was taken to the Operation Theatre. Two 16G Canula was opened on both arms. Right radial artery was canulated and right internal vein canulated to record the blood pressure and CVP.

@Nepalese Heart Journal. Nepalese Heart Journal retains copyright and work is simultaneously licensed under Creative Commons Attribution License CC - BY 4.0 that allows others to share the work with an acknowledgement of the work's authorship and initial publication in this journal. 
Blood pressure was 16 to $180 \mathrm{mmHg}$ systolic and 88 to 96 $\mathrm{mmHg}$ diastolic. Heart rate was 120 to $132 \mathrm{bpm}$.

Introduction was done with fentanyl. Propofol and vecuronium. There was no complication during induction.

Maintenance of anesthesia was done with propofol and fentanyl infusion and $0.5-0.7 \%$ halothane with oxygen. Beta blocker was given to reduce the heart rate. GTN infusion was ready. Vecuronium was given intermittently.

Temperature was recorded by nasopharyngeal probe. Blood gas and saturation was within normal limit, and cardiopulmonary bypass was established by femoro- femoral approach. Chest was opened, about $1200 \mathrm{ml}$ of blood was drained, the abdominal content was reduced and diaphragm repaired.

There was hematoma around the aorta. Cardio pulmonary bypass was started. Mild hypothermia was maintained during pump (up to 34 degree Celsius). Aorta was dissected; clamp was applied in the aorta including the left subclavien artery and distal clamp was applied 2.5 inches below the proximal clamp. Methyl prednisolone $1 \mathrm{gm}$ and $250 \mathrm{ml}$ of $20 \%$ manitol, $6 \mathrm{gm}$ of TEA was given before pump started.

About 2 inches of the ruptured aorta was excised and graft was sown end to end. Pressure was maintained with GTN infusion 4 to $8 \mathrm{mcg} / \mathrm{kg} / \mathrm{minute}$ and main arterial pressure was around $70 \mathrm{mmHg}$ during pump. Temperature rose to normal and de clamaping ewas done. Hemostasis was maintained. Pump was stopped. 3 unit of FFP, 3 unit of RCC and 4 unit of platelet were transfused. Vital were normal and the patient was transferred to the SICU and ventilated overnight with the sedation of propofol and fentanyl. He was extubated next morning. Vitals were normal and he could move all limbs, but he developed abnormal mental behavior and was prescribed haloperidol for 3 days. He was discharged from the SICU on the fifth day of operation.

\section{DISCUSSION}

\section{Preoperative management patient assessment}

Majority of the patients with blunt trauma of the chest have multiple injuries like head injury, trauma in the abdominal viscera, pelvic fracture or fracture of the cervical spine. They are frequently Hypovolumic, hypothermic, hypoxic and acidotic.

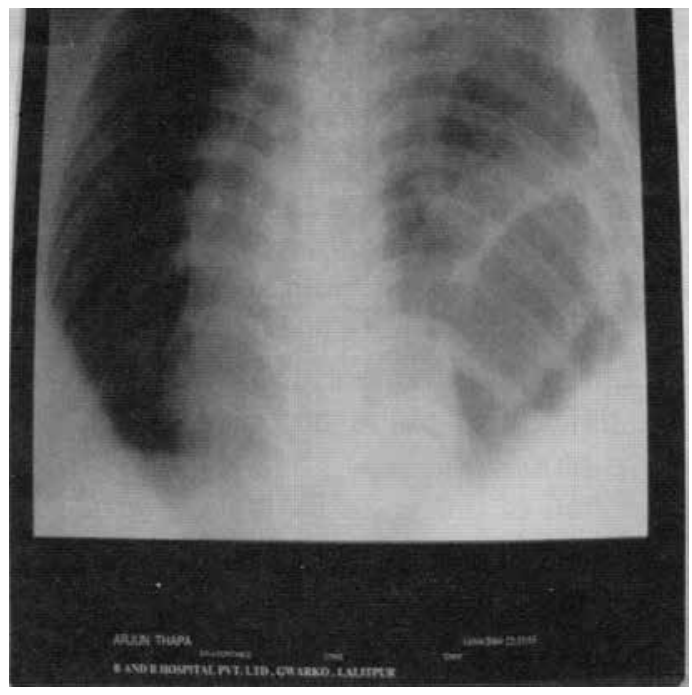

Figure 3: Chest X-Ray showing rupture of the left diaphragm
Hypoxic may be due to airway obstruction, because of trauma, bleeding in the airway, pulmonary confusion, tension pneumothorax, or due to cardiogenic shock and pulmonary edema.

Hypovolumia, acidosis and hypothermia may be due to external bleeding or concealed internal bleeding. With the initial management following the principle of advanced trauma life support, and patients should be investigared accordingly during the process of advanced trauma life support.

After priomary stablilization of the trauma victim, investigarions carried out . chest $\mathrm{x}$-ray provides information which guides to further investigation like, CT and contrast CT.ECG, which will expose any cardiac trauma and guide for further investigation like echocardiography or angiography by which we can detect the condition of the heart, like rupture of aorta, heart valves, trauma in the coronaries and great vessels.

During the process, blood parameter coagulation profiles and blood chemistry are obtained and the essential blood and blood products are managed.

\section{Anesthetic management}

Cardio respiratory insufficiency, tissue hypoxia, acidosis, are the common presentation of this type of victim. After initial management advanced trauma life support principle should be followed.

Pre-oxygenation with $100 \%$ oxygen for 3 to 5 minutes and securing of the airway with rapid sequence intubation, and maintenance of cervical spine immobilization is very important. If difficult airway is suspected then follow American society of anesthesiologist difficult airway algorithm,

If hypoxia is increasing and there is difficulty in ventilation then tension pneumothorax should be considered.

Double lumen endotracheal tube is preferred but it may be difficult to place properly during rapid sequence intubation.

The selection of drugs for intubation is not very important but anesthesiologists should evaluate the situation of the patient's physiological status and ADJUST THE DOSE ACCORDINGLY.

In severely compromised condition patients have maximal sympathetic activity; ketamine may not be a good selection because it can depress the myocardium.

Hypovolumia after induction should promptly be controlled with vasopressure and volume peplacement.

Monitoring should include, SPO2, ETCO2, Artetrial line CVP line, PA catheter, temperature probe, ECG, TEE if needed, awareness monitoring system such as the binspectral index monitoring system is useful.

Anesthetic goal for open aortic surgery is to reduce the stress in the aortic wall, by reducing the blood pressure, systolic less then 140, using beta blocker and GTN.

Aggressive reduction of blood pressure during aortic cross clamping with sodium nitroprusside cand produce postoperative paraplegia.

Duration of aortic cross-clamp is the key determinant of the outcome and the spinal cord protection.

Mild hypothermia (34 degree C) during cross clamp can provide benefit but should re-warm immediately to avoid the coagulopathy.

Femoral artery to femoral vein bypass, left heart to aortic bypass protects the organs like kidney, spinal cord, brain and heart.

Lumbar drain will help to reduce CSF pressure and help to increase the perfusion pressure of the spinal cord. 


\section{Post operative complication}

Pulmonary complication is the most frequent complication. There is pulmonary atelectasis, pneumonia, effusion and pulmonary confusion.

Mediastinal hemorrahage from the repaired side may need reexploration. Due to of edema, hematoma and distorted anatomy will support difficulty to identify recurrent laryngeal nerve can produce vocal cord paralysis. Blood vessels supplying the esophagus can be damaged, which can produce esophageal fistula.

Ischemic damae to spinal cord can occur due to damage to supplying vessel to the spinal cord or prolonged cross clamping time or decreased blood pressure during cross clamp.

Cardiac arrhythmias, like ventricular tachycardia, ventricular fibrillation can occur. Acute renal failure can develop during post operative period.

\section{REFERENCES}

1. Sina L. Moanine, David G. Neschis, et al. Endovascular stenting for traumatic aortic injury: An emerging new standard of care. Ann Thorac Surg 2008;85;1625-30

2. Alan M. Cohen, Jeffery R. crass et al. CT evidence for the "osseous pinch" mechanism of traumatic aortic injury. AJR 1992;159:271-274

3. Edward A. Rittenhouse, david H. Dillard, et al. traumatic Rupture of the Thoracic aorta, Annals of Surgery 1969; Volume 170 , no 1

4. Gilles Godet, Eric Vicaut et al. risk factors for acute postoperative renal failure in thoracic or thoracoabdominal aortic surgery: a prospective study. Anesth Analg 1997;85:1227-32

5. John T. Moloney, Srteven J. Fowler. Anesthetic management of thoracic trauma. Curr Opin Anesthesiol 2008;21:41-46

6. Torazo Wada, Hideki Yao, et al. Prevention and detection spinal cord injury during thoracic and thoracoabdominal aortic repairs. Ann Thorac Surg 2001;72:80-5

7. Hossein Javadpor, John J. O'Toole et al. Traumatic aortic transaction: evidence for the osseous pinch mechanism, Annals of Surgery, 2001.

8. Sung-Min Park, Dae-Hyun Kim et al. triple Aortic Root Injury. Ann Thorac Surg 2009;87:621-3

9. J. Hugh Devitt, Richard F. Mclean et al. Anesthetic management of acute blunt thoracic trauma. Can J Ansesth 1991/38:4/pp506-10

10. Albert T. Cheung, Alberto Pochettino, et al. Safety of lumbar drains in thoracic aortic operations performed with Extracorporeal Circulation. Ann Thorac Surg 2003;76:11907

11. Willam Travis Lau, David K.M. Wong, et al. patent Ductus Arteriosus Masquerading as Aortic Transection in a Trauma victim. Ann thorac 2008;86:e5-6

12. Lorenzo E. Ferri, Paola fata. The current status of traumatic diaphragmatic injury: lessons learned from 105 patients over 13 years. McGill's university Health Center. Division of thoracic surgery, October 24,2007. 